\title{
Microstructures of poly (ethylene glycol) by molding and dewetting
}

\author{
Kahp Y. Suh and Robert Langer ${ }^{\text {a) }}$ \\ Department of Chemical Engineering, Massachusetts Institute of Technology, Cambridge, \\ Massachusetts 02139
}

(Received 24 April 2003; accepted 24 June 2003)

\begin{abstract}
We report on the fabrication of microstructures of poly (ethylene glycol) (PEG) using a soft molding technique. When a patterned poly (dimethylsiloxane) stamp is placed on a wet PEG film, the polymer in contact with the stamp spontaneously moves into the void space as a result of capillary action. Three types of microstructures are observed with the substrate surface completely exposed: a negative replica of the stamp, a two-dimensional projection of the simple cubic structure, and a two-dimensional projection of the diamond structure. A molding process is responsible for the first type and a dewetting process for the final two. A phase diagram is constructed based on the effects of molecular weight and concentration, which shows that mobility and confinement play a crucial role in determining the particular type of microstructure obtained. The PEG microstructure could be used as a lithographic resist in fabricating electronic devices and a resistant layer for preventing nonspecific adsorption of proteins or cells. (C) 2003 American Institute of Physics.
\end{abstract}

[DOI: $10.1063 / 1.1604186]$

Molding of polymer melts is the traditional manufacturing technology of plastics ${ }^{1}$ and, in general, has been developed in the form of injection molding, in which a raw plastic material is melted and then injected into a mold through a nozzle by applying pressure. In the early 1990s, a soft, elastomeric stamp like poly (dimethylsiloxane) (PDMS) was introduced as a new element for molding of polymers, precursors and sol gels. ${ }^{2}$ Since then, a number of unconventional, useful molding techniques have been developed such as micromolding in capillaries, ${ }^{3,4}$ solvent-assisted microcontact molding, ${ }^{5}$ microtransfer molding $(\mu \mathrm{TM}),{ }^{6,7}$ replica molding, ${ }^{8}$ capillary force lithography, ${ }^{9,10}$ and soft molding (SM). ${ }^{11,12}$ Of these, SM provides a convenient way to fabricate polymeric microstructures when the polymer is liquid or wet after spin coating. The technique generally consists of three steps: placing a patterned PDMS stamp on the surface of a spincoated polymer film, allowing the stamp to absorb solvent, and then letting the stamp and the substrate remain undisturbed for a period of time.

Poly (ethylene glycol) (PEG) is a nondegradable, hydrophilic polymer that can be cross-linked into hydrogels using photopolymerization reactions by acrylate functional groups. ${ }^{13}$ Although much work has been done with regard to patterning of PEG hydrogels using photolithography, ${ }^{14-16}$ there essentially has been no soft lithographic technique to pattern PEG hydrogels directly on the surface.

Here, we fabricate PEG microstructures using SM technique. The motivation for this study arises from two key features of the system. First, PEG is a hydrophilic polymer such that it has less affinity for PDMS stamp. The contact angle of a low molecular weight PEG $(\mathrm{MW}=300)$ on a fresh PDMS stamp is $\sim 78^{\circ}$, which indicates that capillary rise into the void space of the stamp would be substantially different from that of hydrophobic polymer such as polysty-

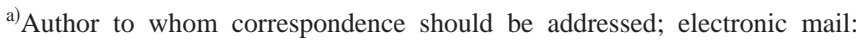
rlanger@mit.edu
}

rene $\left(20^{\circ}-40^{\circ}\right) .^{9,17}$ Therefore, we can expect various PEG microstructures from the competition between capillary force and intermolecular forces at the PEG/PDMS interface. Second, PEG is a well-known polymer for its excellent resistance toward nonspecific adsorption of biological species such as proteins or cells. ${ }^{18}$ If the pattern formation is successful, the PEG microstructure can be used as a general platform for patterning a broad range of biomaterials. In this regard, cross-linking is necessary for low molecular weight PEGs because they are liquid. Without cross-linking, the molded microstructures would be destroyed after peeling off the stamp. For this reason, we used PEG dimethacrylate for cross-linking and the resultant material was cross-linked microstructures immobilized on the surface. Contact angle measurements show that PEG dimethacrylate is slightly hydrophobic $\left(\sim 65^{\circ}\right)$, which does not affect the overall results, however.

PDMS stamps were fabricated by casting PDMS (Sylgard 184 Silicon elastomer, Essex Chemical) against a complementary relief structure prepared by a photolithographic method. To cure, a 1:10 ratio of the curing agent and the pre-polymer were mixed and incubated at $70^{\circ} \mathrm{C}$ for $1 \mathrm{~h}$. PDMS was then detached from the master and cut. The stamp had protruding (positive) boxes with four different lateral dimensions: 3, 5, 7, and $10 \mu \mathrm{m}$ with a constant step height of $1.5 \mu \mathrm{m}$. Silicon dioxide and glass were used as the substrates. Prior to spin coating, the substrates were cleaned by rinsing with acetone and ethanol several times to remove excess organic molecules and dried in a nitrogen gas stream. To investigate the effects of molecular weight (MW), four different molecular weights were tested: 330, 550, 1000, and 4600. For MW of 330 and 550, PEG dimethacrylates were used for cross-linking. Various concentrations of PEG or PEG dimethacrylate solution in methanol $(1,2,5,10,20,50$, 70 , and 100 wt $\%$ or pure PEG dimethacrylate) were prepared. For liquid PEGs, 1 wt \% of the UV initiator (2,2dimethoxy-2-phenylacetophenone) with respect to the 


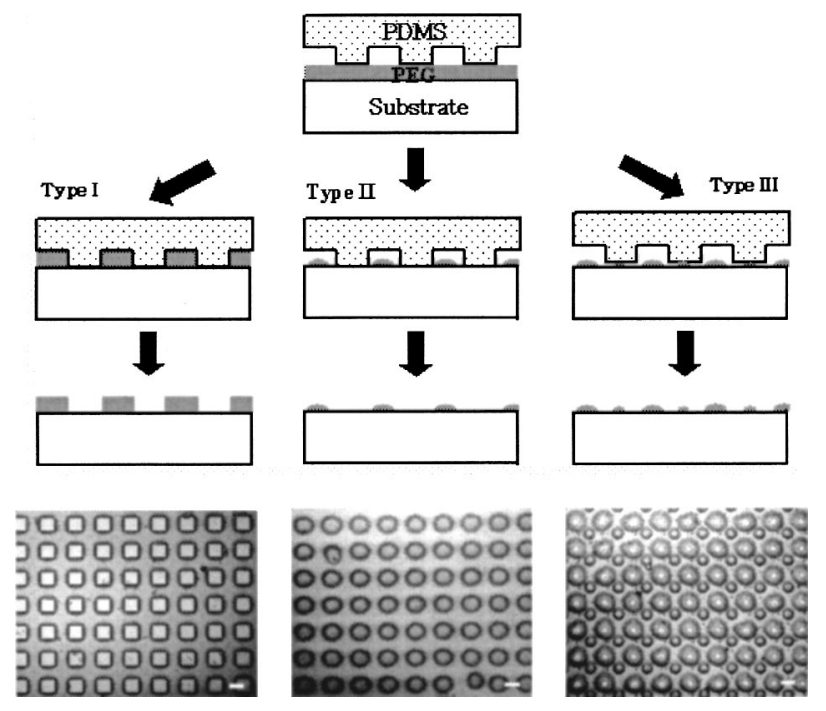

FIG. 1. Schematic diagram of the experimental procedure and three types of PEG microstructures along with the corresponding optical images. A molding process is responsible for the first type and a dewetting process for the final two. Note that no traces of the original stamp are observed in the two dewetting patterns (type II and III) and the substrate surface is completely exposed for all the three types. The scale bar indicates $10 \mu \mathrm{m}$.

amount of polymer was added in the solutions. The polymer was then spin coated (Model CB 15, Headaway Research, Inc.) onto a substrate at $3000 \mathrm{rpm}$ for $10 \mathrm{~s}$. The film thickness ranged from $60 \mathrm{~nm}$ to $1.2 \mu \mathrm{m}$ as measured by ellipsometry. The patterned PDMS stamp was carefully placed onto the surface to make conformal contact and the stamp and the substrate remained undisturbed for a period of time, typically, for $30 \mathrm{~min}$. For liquid PEGs, the samples were placed for $2 \mathrm{~h}$ under a $365 \mathrm{~nm}, 15 \mathrm{~mW} / \mathrm{cm}^{2}$ low-power black-light inspection lamp (ELC-251, Electro-Lite Corp.) for crosslinking.

Figure 1 outlines the procedure and three types of microstructures that are observed in our experiment along with the typical optical images. The structures result from two processes: molding and dewetting. The schematic diagram on the left hand side depicts the molding process, in which a negative replica of the stamp forms on the surface (type I). On the other hand, the two diagrams in the middle and the right hand side illustrate the dewetting process. Such dewetting patterns can be classified into two types. The first pattern resembles the two-dimensional projection of simple cubic structure (type II) and the second the two-dimensional projection of diamond structure (type III). A notable finding in dewetting patterns is that there are no traces of the original PDMS stamp, which indicates that capillary rise is completely suppressed. In comparison to this, capillary rise cannot be suppressed in the case of a hydrophobic polymer (i.e., polystyrene) such that traces of the stamp are always observed on the surface after dewetting. ${ }^{19}$ These dewetting patterns would be useful for templating two-dimensional photonic crystals and stacking into three-dimensional assembly.

The three types of microstructures turn out to be highly dependent on the molecular weight and concentration of PEG, which is clearly shown in a phase diagram in Fig. 2. A $10 \mu \mathrm{m}$ box pattern was used for this phase diagram. As seen from the figure, the molding process dominates throughout Downloaded 19 Aug 2003 to 18.42.2.210. Redistribution subject

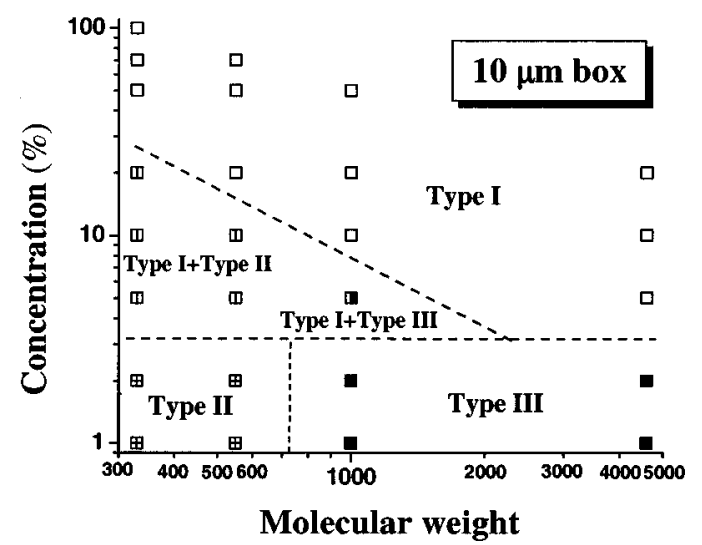

FIG. 2. A phase diagram of PEG microstructure based on the molecular weight and concentration using a $10 \mu \mathrm{m}$ box stamp. Three demarcation lines are present to guide the eyes. In the phase diagram, there is a transition window, in which two structures can coexist (i.e., type I and II) and the window gets smaller as the molecular weight increases.

all the molecular weights as long as the concentration is relatively high. This can be readily understood in that the film thickness would be high enough to fill up the void space of the stamp as the concentration increases. Once the void space is fully taken up, no dewetting can be observed, which may be referred to as the "confinement" effect. On the other hand, the demarcation line between the molding (type I) and the dewetting (type II and type III) is also dependent on the molecular weight such that the tendency toward the molding increases as the molecular weight increases. This result can be attributed to the effects of mobility of PEG, which can be termed as the "mobility" effect. If the molecular weight is relatively low $(<1000)$, the polymer has a large mobility, thereby easily achieving the thermodynamically equilibrium condition (i.e., the dewetting structure in this case). By contrast, the movement of polymer chains will be strongly restricted under the confinement of the stamp if the molecular weight is relatively high. This argument can be further supported by another boundary line between type II and type III structures in the bottom region in Fig. 2. The fact that the type III structure dominates for high molecular weight reveals that small polymer islands are frozen during the molding process due to the confinement and they are observed after the stamp removal. In the phase diagram, there is a transition region, in which the molded and dewetted structures can coexist. The transition window appears to get smaller as the molecular weight increases. If the pattern size goes smaller, i.e., less than $10 \mu \mathrm{m}$, a type I structure can be seen at lower concentrations (the demarcation line moves downward) and a type II structure at higher molecular weights (the demarcation line moves right).

The molded and dewetted PEG microstructures provide a number of potentially useful technological applications since the substrate surface is completely exposed for all the three types of structures. With regard to optical lithography or photolithography, this is of major interest because a residual layer is a problem for most molding techniques. ${ }^{2,9,11}$ If the surface can be exposed, there is essentially no difference between the molding and photolithography in that the molding step completely replaces the exposure and developing steps in photolithography. In addition, the patterned PEG sur-
o AlP license or copyright, see http://ojps.aip.org/aplo/aplcr.jsp 

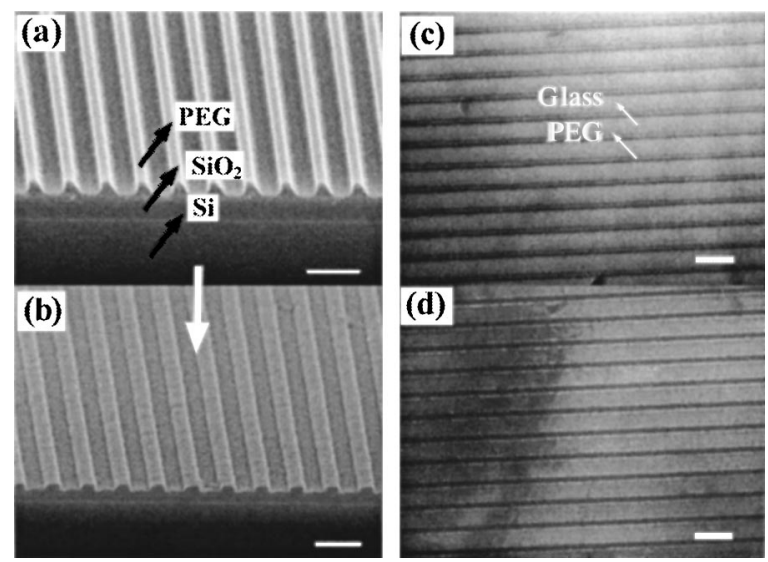

FIG. 3. SEM images of a $700 \mathrm{~nm}$ PEG pattern (a) and the corresponding substrate structure (b) after pattern transfer using RIE. The PEG structure was successfully transferred such that well-defined $\mathrm{SiO}_{2}$ lines are observed. The scale bar indicates $2 \mu \mathrm{m}$. (c) An optical and (d) a fluorescent image of FITC-BSA adsorbed PEG pattern on glass substrate (5 $\mu \mathrm{m}$ lines). Most BSAs adsorbed on the exposed glass surface, not on the PEG surface, which provides sharp contrast in the fluorescent image. The scale bar indicates 10 $\mu \mathrm{m}$.

face can be directly used as a resistant layer to prevent nonspecific adsorption of proteins or cells.

To verify the effectiveness as a resist, the transfer of the polymer pattern to the underlying substrate was carried out using reactive ion etching (RIE) in one step with $\mathrm{CH}_{3}$ (40 $\mathrm{sccm}) / \mathrm{CF}_{4}(10 \mathrm{sccm})$ plasma at a pressure of $50 \mathrm{mTorr}$ and a power of $125 \mathrm{~W}$. The etch rate of $\mathrm{SiO}_{2}$ is about five times higher than that of polymer. After the etching is completed, the residual polymer is removed by methanol and the resulting structures were examined by scanning electron microscopy (SEM). Figures 3(a) and 3(b) show the SEM micrographs of a PEG pattern (a) and the corresponding substrate structure after pattern transfer (b). The pattern transfer turned out to be successful so that well-defined $\mathrm{SiO}_{2}$ structures (700 $\mathrm{nm}$ lines) could be obtained. The only shortcoming of the PEG pattern is a lack of edge definition possibly due to the large surface tension of PEG $(\sim 43 \mathrm{mN} / \mathrm{m})^{18}$ and the incomplete wetting, which needs to be addressed for smaller feature sizes. If the edge profile and etching conditions are tailored to meet the stringent pattern fidelity, the molding step could replace the current photolithographic process in fabricating simple electronic devices.

To examine the effectiveness of the PEG pattern and surfaces to reduce protein adsorption, we prepared fluorescein isothiocyanate-labeled bovine serum albumin (FITCBSA). FITC-BSA was dissolved in $10 \mathrm{mM}$ phosphate buffered saline (PBS) solution $(p \mathrm{H}=7.4)$. The PEG pattern was prepared on glass substrate and the samples were immersed in the solution for $30 \mathrm{~min}$. After rinsing with PBS solution and water several times and drying in a stream of nitrogen, the surface was imaged using an inverted microscope (Axiovert 200, Zeiss). Figures 3(c) and 3(d) show $5 \mu \mathrm{m}$ lines of
PEG pattern on glass substrate (c) and the corresponding fluorescent image (d). As expected, most BSAs deposit on the exposed glass surface, which shows a spatially welldefined protein pattern. This indicates that the cured PEG film also provides protein resistance as with PEG terminated self-assembled monolayers. ${ }^{20}$ Although not shown, the protein pattern could be reduced below $1 \mu \mathrm{m}$ with suitably prepared PDMS stamps over large areas.

In summary, we have fabricated PEG microstructures using the soft molding technique and tested their effectiveness as a resist for pattern transfer and a resistant layer for the adhesion of proteins. Three types of microstructures are observed depending on the competition between molding and dewetting process. To elucidate the competition, a phase diagram is constructed based on the effects of molecular weight and concentration, which reveals that confinement and mobility can account for the complex behavior of PEG polymer within the PDMS stamp. The PEG microstructures presented here could provide a new way of fabricating simple electronic and biological devices and studying dewetting behavior in a repulsive environment.

This research was supported by, or supported in part by, the U.S. Army through the Institute for Soldier Nanotechnologies, under Contract No. DAAD-19-02-D0002 with the U.S. Army Research Office.

${ }^{1}$ D. V. Rosato, in Injection Molding Handbook, 1st ed., edited by D. V. Rosato (Van Nostrand Reinhold, New York, 1986), p. 3.

${ }^{2}$ Y. N. Xia, J. A. Rogers, K. E. Paul, and G. M. Whitesides, Chem. Rev. (Washington, D.C.) 99, 1823 (1999).

${ }^{3}$ E. Kim, Y. Xia, and G. M. Whitesides, Nature (London) 376, 581 (1995).

${ }^{4}$ J. A. Rogers, Z. N. Bao, and V. R. Raju, Appl. Phys. Lett. 72, 2716 (1998).

${ }^{5}$ E. Kim, Y. Xia, X. M. Zhao, and G. M. Whitesides, Adv. Mater. (Weinheim, Ger.) 9, 651 (1997)

${ }^{6}$ X. M. Zhao, Y. Xia, and G. M. Whitesides, Adv. Mater. (Weinheim, Ger.) 8, 837 (1996).

${ }^{7}$ T. Deng, M. Prentiss, and G. M. Whitesides, Appl. Phys. Lett. 80, 461 (2002).

${ }^{8}$ Y. Xia, E. Kim, X. M. Zhao, J. A. Rogers, M. Prentiss, and G. M. Whitesides, Science 273, 347 (1996).

${ }^{9}$ K. Y. Suh, Y. S. Kim, and H. H. Lee, Adv. Mater. (Weinheim, Ger.) 13, 1386 (2001)

${ }^{10}$ G. Tae, R. G. H. Lammertink, J. A. Kornfield, and J. A. Hubbell, Adv. Mater. (Weinheim, Ger.) 15, 66 (2003).

${ }^{11}$ Y. S. Kim, K. Y. Suh, and H. H. Lee, Appl. Phys. Lett. 79, 2285 (2001).

${ }^{12}$ Y. S. Kim, J. Park, and H. H. Lee, Appl. Phys. Lett. 81, 1011 (2002).

${ }^{13}$ A. S. Sawhney, C. P. Pathak, and J. A. Hubbell, Macromolecules 26, 581 (1993).

${ }^{14}$ M. J. Lesho and N. F. Sheppard, Sens. Actuators B 37, 61 (1996).

${ }^{15}$ D. J. Beebe, J. S. Moore, J. M. Bauer, Q. Yu, R. H. Liu, C. Devadoss, and B. H. Jo, Nature (London) 404, 588 (2000).

${ }^{16}$ A. Revzin, R. J. Russell, V. K. Yadavalli, W.-G. Koh, C. Deister, D. D. Hile, M. B. Mellott, and M. V. Pishko, Langmuir 17, 5440 (2001).

${ }^{17}$ G. Reiter, Phys. Rev. Lett. 68, 75 (1992).

${ }^{18}$ J. M. Harris and S. Zalipsky, Poly (ethylene Glycol): Chemistry and Biological Applications (American Chemical Society, Washington, DC, 1997).

${ }^{19}$ K. Y. Suh, J. Park, and H. H. Lee, J. Chem. Phys. 116, 7714 (2002).

${ }^{20}$ G. M. Whitesides, E. Ostuni, S. Takayama, X. Jiang, and D. E. Ingber, Annu. Rev. Biomed. Eng. 3, 335 (2001). 